\title{
Compact Microstrip Coupled-Line Bandpass Filter With Two Cross-Couplings for Creating Multiple Transmission Zeros
}

\author{
Chao-Huang Wu, Yo-Shen Lin, Chi-Hsueh Wang, and Chun Hsiung Chen \\ Department of Electrical Engineering and Graduate Institute of Communication Engineering, \\ National Taiwan University, Taipei 106, Taiwan. \\ (email: chchen@ew.ee.ntu.edu.tw)
}

\begin{abstract}
A compact coupled-line bandpass filter with two capacitively cross-coupled paths to create multiple transmission zeros is proposed. The locations of transmission zeros can be adjusted by varying the values of cross-coupled capacitances so as to improve the filter selectivity. Specifically, a 4th-order microstrip coupled-line bandpass filter centered at $4.9 \mathrm{GHz}$ with a 3-dB bandwidth of $6 \%$ and six transmission zeros is implemented and examined.
\end{abstract}

\section{INTRODUCTION}

In microwave communication system design, a filter with compact size, low insertion loss, and good selectivity is usually needed to reduce the cost and to enhance the system performance. In order to achieve better selectivity, many improvements on creating suitable transmission zeros were reported. In [1]-[2], the filters with a crosscoupling between resonators to generate transmission zeros were examined. Multiple transmission zeros were obtained by using suitable input, output, and interstage tapped-couplings [3]. In [4]-[5], quarter-wavelength $(\lambda / 4)$ open stubs and capactively coupled gaps were introduced in the conventional coupled-line filter structure to create the transmission zeros at stopband. The trisection and quadruplet microstrip bandpass filters [6]-[7] based on folded $\lambda / 4$ resonators were proposed to achieve very compact circuit sizes with one or two transmission zeros. The compact hairpin filter with asymmetric tapping feed lines to produce transmission zeros was also discussed in [8]-[9]. In our previous works [10]-[12], by introducing a capacitive cross-coupling effect, two transmission zeros at upper and lower stopbands may be created for improving the filter selectivity.

In this study, the 4th-order microstrip bandpass filter discussed in [13] and shown in Fig. 1 is extended to develop a novel filter structure as shown in Fig. 2 which has two capacitive cross-couplings to create multiple transmission zeros. Comparing with the basic 4th-order bandpass filter in Fig. 1 which has a cross-coupled path provided by the capacitance $\mathrm{C}_{2}$, the proposed filter has an additional cross-coupled capacitance $\mathrm{C}_{3}$ introduced directly between the input and output ports as shown in Fig. 2. Therefore, the new filter structure may create multiple transmission zeros at the upper and lower stopbands such that the selectivity of the proposed filter may further be improved while keeping the same circuit size.

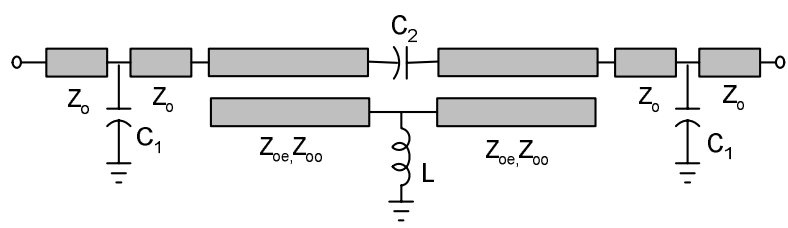

Fig. 1. Circuit model of the 4th-order bandpass filter in [13] with a capacitively cross-coupled path to create two transmission zeros.

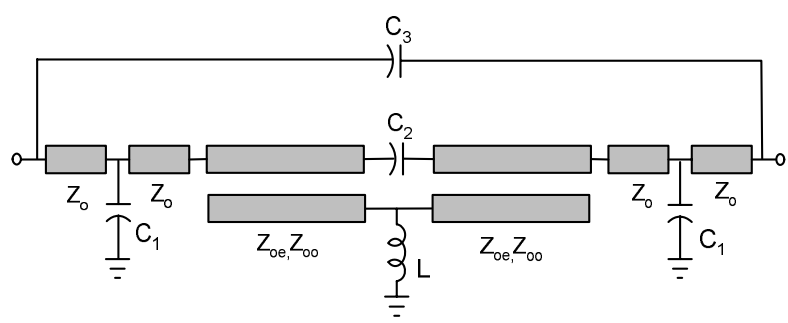

Fig. 2. Circuit model of the proposed 4th-order bandpass filter with two capacitively cross-coupled paths to create multiple transmission zeros.

\section{FILTER STRUCTURE AND IMPLEMENTATION}

Fig. 2 shows the proposed filter circuit model with two capacitively cross-coupled paths to create multiple transmission zeros. By neglecting the cross-coupling effects, i.e. $\mathrm{C}_{2}=\mathrm{C}_{3}=0$, the coupled-line section with open circuited terminal may be equivalent to a $\mathrm{J}$ inverter along with two transmission lines for narrow band [14] and the shunt capacitor $\mathrm{C}_{1}$ or inductor $\mathrm{L}$ with two transmission line sections at its two ends may be equivalent to a $\mathrm{K}$ inverter [15]. Thus, the equivalent circuit model is the same as the one in [13].

Based on the circuit model in Fig. 2, a novel 4th-order microstrip coupled-line bandpass filter is implemented as in Fig. 3 which has two cross-coupled capacitors $C_{2}$ and $\mathrm{C}_{3}$ to generate four to six transmission zeros depending on the values of capacitances $\mathrm{C}_{2}$ and $\mathrm{C}_{3}$. Here, the crosscoupled capacitor $\mathrm{C}_{2}$ is realized by the gap-coupled configuration between the open-ends of two coupled-line sections. In order to achieve the desired amount of crosscoupling through the capacitor $\mathrm{C}_{3}$, the two coupled-lines are bended by an angle $\theta_{1}$ and the transmission-line sections by an angle $\theta_{2}$. The shunt capacitors $C_{1}$ are 
Port 1

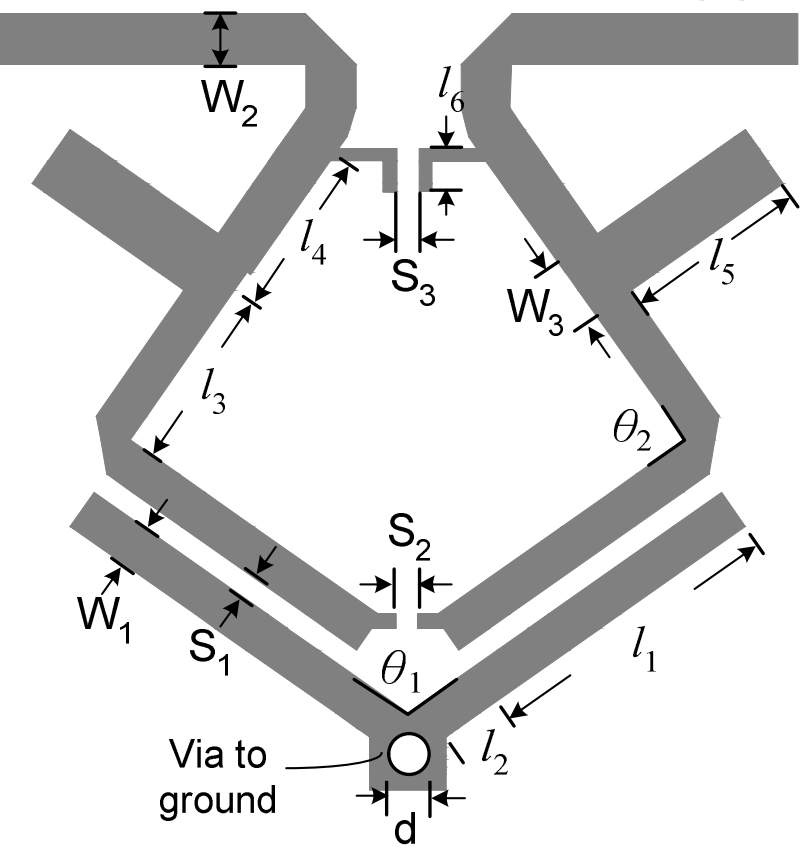

Fig. 3. Layout of the proposed 4th-order microstrip coupledline bandpass filter with two capacitive cross-couplings to create multiple transmission zeros. $\left(\mathrm{W}_{1}=0.97 \mathrm{~mm}, \mathrm{~S}_{1}=0.5 \mathrm{~mm}\right.$, $\mathrm{W}_{2}=1.17 \mathrm{~mm}, \mathrm{~S}_{2}=0.3 \mathrm{~mm}, \mathrm{~W}_{3}=1.53 \mathrm{~mm}, \mathrm{~S}_{3}=0.15 \mathrm{~mm}, l_{1}=7.08 \mathrm{~mm}$ $l_{2}=1.4 \mathrm{~mm}, l_{3}=4.3 \mathrm{~mm}, l_{4}=4.2 \mathrm{~mm}, l_{5}=4.3 \mathrm{~mm}, l_{6}=0.8 \mathrm{~mm}, \theta_{1}=70^{\circ}$, $\theta_{2}=90^{\circ}$, and $\mathrm{d}=1.1 \mathrm{~mm}$.)

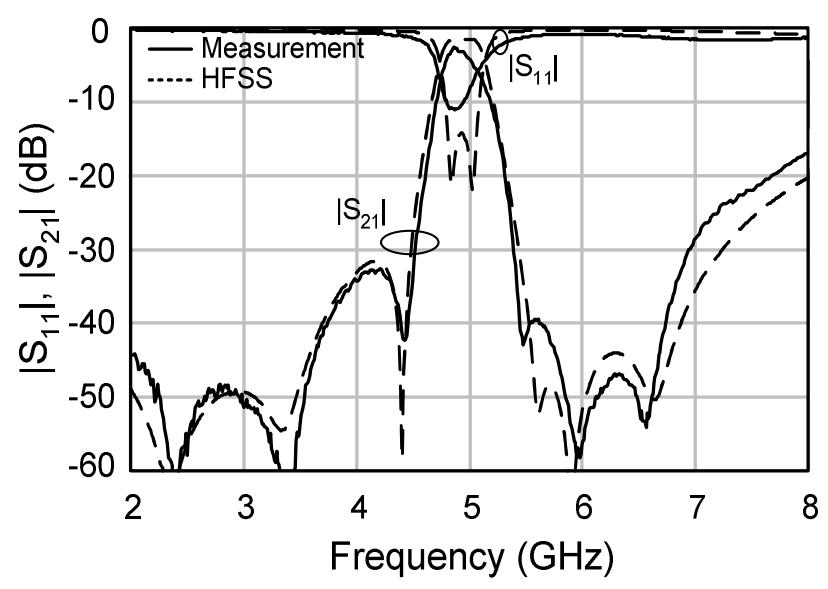

Fig. 4. Measured and simulated results for the proposed 4thorder microstrip coupled-line bandpass filter shown in Fig. 3.

accomplished by the open stubs and the inductor $\mathrm{L}$ is realized by the via hole so as to fit the values of the $\mathrm{K}$ inverters.

The proposed filter structure is implemented using the microstrip configuration, and is fabricated on a Rogers RO4003c substrate $\left(\varepsilon_{\mathrm{r}}=3.38, \tan \delta=0.0023\right.$, and thickness $\mathrm{h}=0.508 \mathrm{~mm}$ ). The simulated results by the fullwave simulator HFSS and the measured ones for the filter in Fig. 3 are shown in Fig. 4. The measured center frequency is at $4.9 \mathrm{GHz}$. The minimum measured insertion loss is $2.5 \mathrm{~dB}$ at $4.87 \mathrm{GHz}$, and the $3-\mathrm{dB}$ bandwidth is $6 \%$. Six transmission zeros at $2.38 \mathrm{GHz}$, $3.34 \mathrm{GHz}, 4.33 \mathrm{GHz}, 5.39 \mathrm{GHz}, 5.89 \mathrm{GHz}$, and $6.48 \mathrm{GHz}$

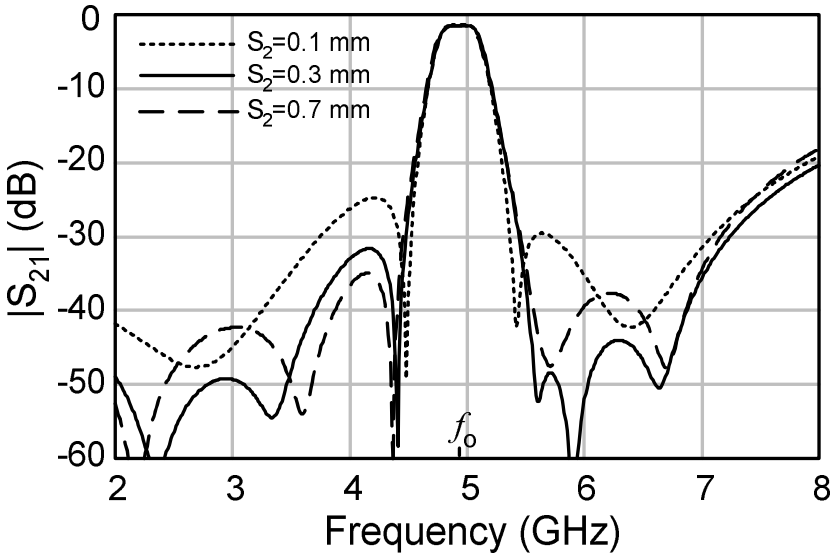

(a)

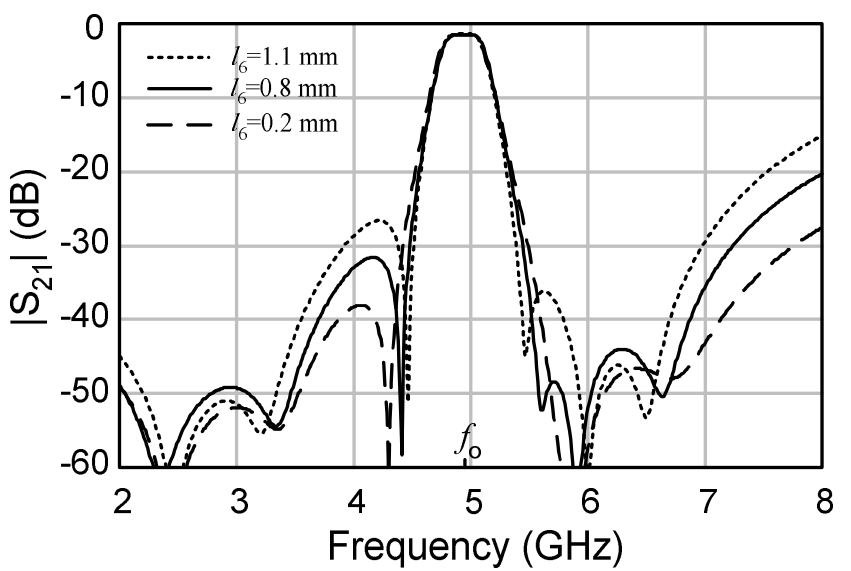

(b)

Fig. 5. Full-wave simulated responses of the proposed 4th-order microstrip filter in Fig. 3 for (a) various values of $S_{2}$ to control the values of capacitance $C_{2}$, (b) various values of $l_{6}$ to control the values of capacitance $\mathrm{C}_{3}$.

are observed as expected. Note that the inclusion of the cross-coupled capacitor $\mathrm{C}_{3}$ has created the multiple transmission zeros to improve the filter selectivity but at the expense of degrading the stopband rejection level.

\section{TRANSMISSION ZEROS}

The locations of transmission zeros for the proposed filter may actually be adjusted by varying the parameter $\mathrm{S}_{2}$ (Fig. 3) to control the value of $\mathrm{C}_{2}$ and the parameters $l_{6}$ and $\mathrm{S}_{3}$ to control that of $\mathrm{C}_{3}$. Shown in Fig. 5 are the fullwave simulated responses of the proposed filter with $\mathrm{S}_{2}$ and $l_{6}$ in Fig. 3 as parameters. As shown in Fig. 5(a), the two left-most transmission zeros (with respect to the passband center frequency $f_{\mathrm{o}}$ ) will move and close to each other and the transmission zeros at upper stopband will move toward the passband edge as the parameter $S_{2}$ is decreased to increase the value of $\mathrm{C}_{2}$. For the case $\mathrm{S}_{2}=$ $0.1 \mathrm{~mm}$, only four transmission zeros are observed because some of transmission zeros would be too close to be distinguishable. As shown in Fig. 5(b), the inner pair of transmission zeros will move toward the passband edge and the stopband rejection level will degrade as the 


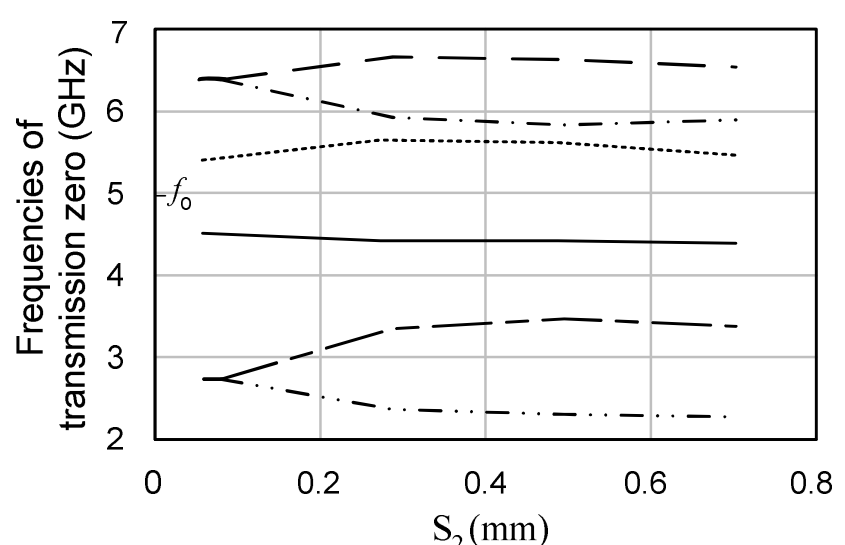

(a)

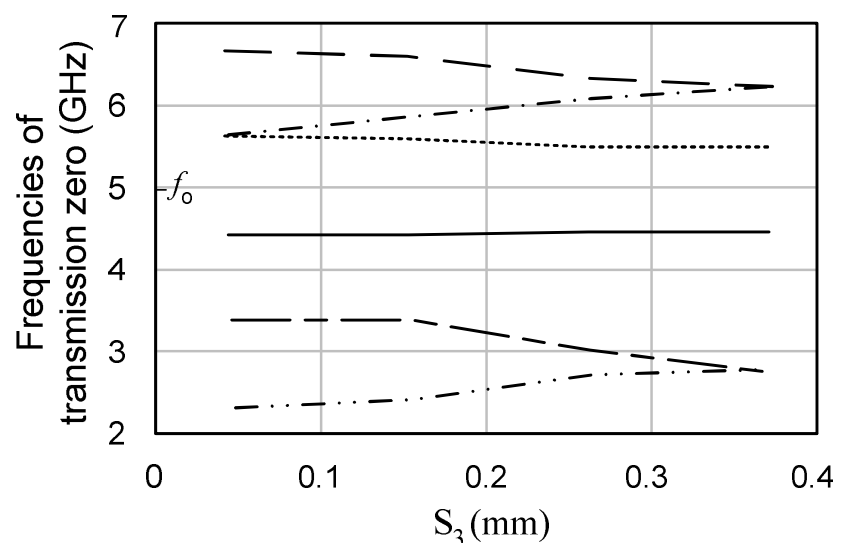

(b)

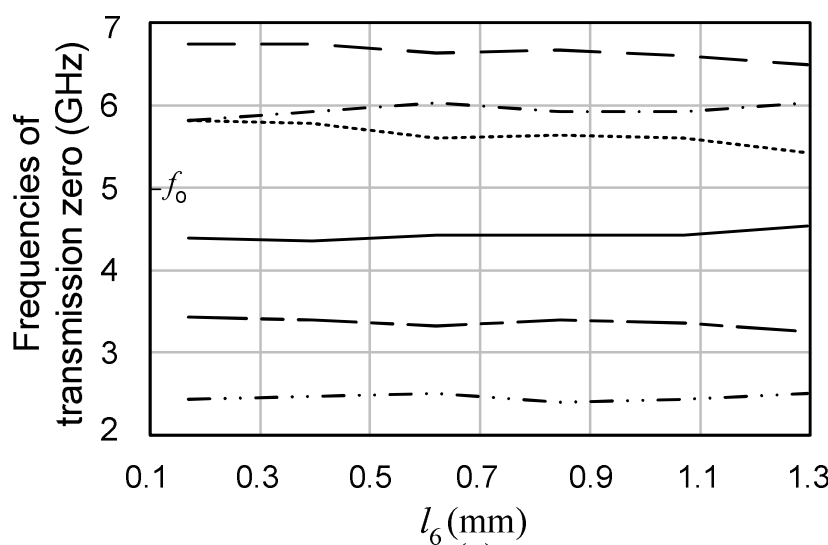

(c)

Fig. 6. Full-wave simulated curves to relate the frequencies of transmission zeros to the parameters (a) $\mathrm{S}_{2}\left(l_{6}=0.8 \mathrm{~mm}, \mathrm{~S}_{3}=\right.$ $0.15 \mathrm{~mm})$, (b) $\mathrm{S}_{3}\left(l_{6}=0.8 \mathrm{~mm}, \mathrm{~S}_{2}=0.3 \mathrm{~mm}\right)$, and (c) $l_{6}\left(\mathrm{~S}_{2}=\right.$ $0.3 \mathrm{~mm}, \mathrm{~S}_{3}=0.15 \mathrm{~mm}$ ) in Fig. 3 .

parameter $l_{6}$ is increased to increase the value of $\mathrm{C}_{3}$. For the case $l_{6}=0.2 \mathrm{~mm}$, the frequency response only has five transmission zeros. Therefore, according to the movement tendency of transmission zeros in Fig. 5, the values of $\mathrm{C}_{2}$ and $\mathrm{C}_{3}$ can be determined for the desired locations of the transmission zeros so as to achieve the required fall-off rate at the passband edge and the desired level of stopband rejection.

Shown in Fig. 6 are the detail full-wave simulated curves to relate the frequencies of the transmission zeros to the parameters $\mathrm{S}_{2}, \mathrm{~S}_{3}$, and $l_{6}$. Comparing Fig. 6(b) with Fig. 6(c), the parameter $\mathrm{S}_{3}$ has more influence than $l_{6}$ on the frequencies of transmission zeros. The parameter $\mathrm{S}_{3}$ can first be selected and the parameter $l_{6}$ is then tuned to fit the specification. Some transmission zeros would be too close to be distinguishable as the parameter $S_{3}$ is increased or decreased. Note that the frequencies of transmission zeros might slightly be changed because of the dimensions variation in the fabrication process.

\section{CONCLUSION}

In this work, a compact 4th-order microstrip bandpass filter with multiple transmission zeros has been proposed. By introducing two capacitively cross-coupled paths, the proposed filter exhibits multiple transmission zeros at upper and lower stopbands. The locations of these transmission zeros may simply be adjusted by varying the values of cross-coupled capacitances. The proposed bandpass filter is useful for application in the communication systems when better selectivity and good stopband rejection are required.

\section{ACKNOWLEDGEMENT}

This work was supported by the National Science Council of Taiwan under Grant NSC 93-2752-E-002-001PAE and Grant NSC 93-2219-E-002-021.

\section{REFERENCES}

[1] R. Levy, "Filters with single transmission zeros at real or imaginary frequencies," IEEE Trans. Microwave Theory Tech., vol. 24, pp. 172-181, Apr. 1976.

[2] K. T. Jokela, "Narrow-band stripline or microstrip filters with transmission zeros at real and imaginary frequencies," IEEE Trans. Microwave Theory Tech., vol. 28, pp. 542-547, June. 1980.

[3] K. Wada, and I. Awai, "Heuristic models of halfwavelength resonator bandpass filter with attenuation poles," Electron. Lett., pp. 400-401, March 1999.

[4] J.-R. Lee, J.-H. Cho, and S.-W. Yun, "New compact bandpass filter using microstrip $\lambda / 4$ resonators with open stub inverter," IEEE Microwave Guided Wave Lett., vol. 10, pp. 526-527, Dec. 2000.

[5] Y.-M. Yan, Y.-T. Chang, H. Wang, R.-B. Wu, and C. H. Chen, "Highly selective microstrip bandpass filters in $\mathrm{Ka}$ band," in 32th European Microwave Conf. Proc., pp. 1137-1140, 2002.

[6] C.-Y. Chang, C.-C. Chen, and H.-J. Huang, "Folded quarter-wave resonator filters with Chebyshev, flat group delay, or quasi-elliptic function response," in IEEE MTTS Int. Microwave Symp. Dig., 2002, pp.1609-1612.

[7] C.-Y. Chang and C.-C. Chen, "A novel coupling structure suitable for cross-coupled filters with folded quarter-wave resonators," IEEE Microwave Wireless Comp. Lett., vol. 13, pp. 517-519, Dec. 2003. 
[8] C.-M. Tsai, S.-Y Lee, and C-C Tsai, "Performance of a planar filter using a $0^{\circ}$ feed structure," IEEE Trans. Microwave Theory Tech., vol. 50, pp. 2362-2367, Oct. 2002.

[9] L.-H. Hsieh and K. Chang, "Tunable microstrip bandpass filters with two transmission zeros," IEEE Trans. Microwave Theory Tech., vol. 51, pp. 520-525, Feb. 2003.

[10] Y.-S. Lin and C. H. Chen, "Novel balanced microstrip coupled-line bandpass filters," in URSI Int. Symp. on Electromagnetic Theory, 2004, pp. 567-569.

[11] C.-H. Wang, Y.-S. Lin, and C. H. Chen, "Novel inductance-incorporated microstrip coupled-line bandpass filters with two attenuation poles," in IEEE MTT-S Int. Microwave Symp., Dig., 2004, pp.1979-1982.
[12] Y.-S. Lin, C.-H. Wang, C.-H. Wu and C. H. Chen, "Novel compact parallel-coupled microstrip bandpass filters with lumped-element K-inverters," to appear in IEEE Trans. Microwave Theory Tech.

[13] Y.-S. Lin, H.-M. Yang, C. H. Chen, "Miniature microstrip parallel-coupled bandpass filters based on lumped-distributed coupled-line sections," to appear in 2005 IEEE MTT-S Int. Microwave Symp. Dig.

[14] S. B. Cohn, "Parallel-coupled transmission-lineresonator filters," IRE Trans. Microwave Theory Tech., vol. 6, pp. 223-231, April 1958.

[15] G. L. Mattaei, L. Young, and E. M. T. Jones, Microwave Filters, Impedance-Matching Networks, and Coupling Structures. Norwood, MA: Artech House, 1980. 